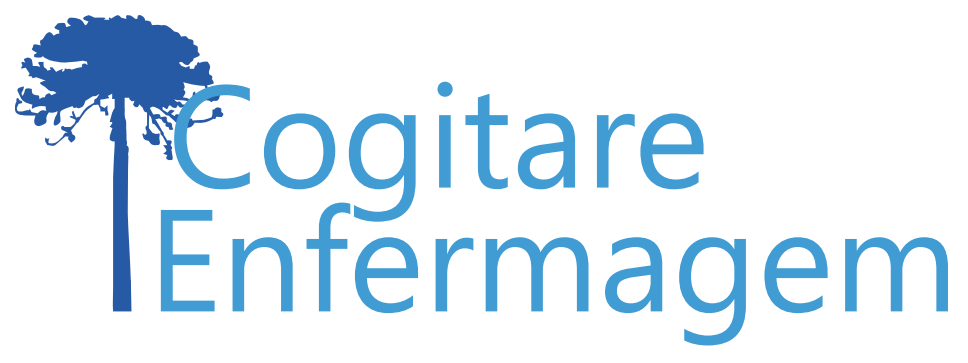

\title{
ANÁLISE BIBLIOMÉTRICA DE ARTIGOS SOBRE CATETERIZAÇÃO VENOSA PERIFÉRICA EM PEDIATRIA
}

Viviane Reis Fontes da Silva1, Isabella Barbosa Meireles², César Cheng ${ }^{3}$, Roberto Carlos Lyra da Silva ${ }^{4}$, Carlos Roberto Lyra da Silva5 ${ }^{5}$ Luiz Carlos Santiago ${ }^{6}$

\section{RESUMO}

Objetivo: analisar a produção intelectual sobre cateterização venosa periférica pediátrica, disponibilizada na base Scopus.

Metodo: estudo bibliométrico que abordou a produção registrada eletronicamente de 1969 a 2018, com análise estatística descritiva.

Resultados: foram recuperados 213 artigos publicados em 123 periódicos. A Enfermagem contribuiu com $21(9,8 \%)$ artigos da área de Saúde. Os Estados Unidos lideraram com 92 artigos (43,1\%). O estudo mostra as limitações da estratificação de periódicos pelo WebQualis ao demonstrar que 41 $(33,36 \%)$ periódicos não constam em nenhum estrato Qualis.

Conclusão: os dados elaborados por meio estudo mensuraram a contribuição do conhecimento científico derivado das publicações acerca da cateterização. Portanto, seus indicadores podem ser úteis para o planejamento e a execução de novas pesquisas, e para o conhecimento da comunidade cientifica sobre o sistema em que está inserida.

DESCRITORES: Enfermagem; Bibliometria; Fator de Impacto; Cateterismo Periférico; Pediatria.

COMO REFERENCIAR ESTE ARTIGO:

Silva VRF da, Meireles IB, Cheng C, Silva RCL da, Silva CRL da, Santiago LC. Análise bibliométrica de artigos sobre cateterização venosa periférica em pediatria. Cogitare enferm. [Internet]. 2019 [acesso em "colocar data de acesso, dia, mês abreviado e ano"]; 24. Disponível em: http://dx.doi.org/10.5380/ce.v24i0.59232.

Este obra está licenciado com uma Licença Creative Commons Atribuição 4.0 Internacional.

${ }^{1}$ Enfermeira. Mestranda em Tecnologia no Espaço Hospitalar. Enfermeira da Unidade de Pediatria do Hospital Municipal Souza Aguiar. Rio de Janeiro, RJ, Brasil. (-)

${ }^{2}$ Enfermeira. Mestranda em Tecnologia no Espaço Hospitalar. Enfermeira do Núcleo de Segurança do Paciente do Hospital Cardoso Fontes. Rio de Janeiro, RJ, Brasil. 9

${ }^{3}$ Engenheiro da Informação. Doutorando em Enfermagem e Biociência. Engenheiro do Instituto Nacional do Câncer. Rio de Janeiro, RJ, Brasil. Contribuição: análise de dados do estudo. (P)

${ }^{4}$ Enfermeiro. Doutor em Enfermagem. Coordenador do Programa de Pós-Graduação em Enfermagem e Biociências da Universidade Federal do Estado do Rio de Janeiro. Rio de Janeiro, RJ, Brasil. ()

${ }^{5}$ Enfermeiro. Doutor em Enfermagem. Docente do Programa de Pós-Graduação em Enfermagem e Biociências da Universidade Federal do Estado do Rio de Janeiro. Rio de Janeiro, RJ, Brasil. 9

${ }^{6}$ Enfermeiro. Doutor em Enfermagem. Docente do Programa de Pós-Graduação em Enfermagem e Biociências da Universidade Federal do Estado do Rio de Janeiro. Rio de Janeiro, RJ, Brasil. 


\title{
BIBLIOMETRIC ANALYSIS OF ARTICLES ABOUT PERIPHERAL VENOUS CATHETERIZATION IN PEDIATRICS
}

\author{
ABSTRACT \\ Objective: analyze the intellectual production on pediatric peripheral venous catheterization \\ available in the database Scopus. \\ Method: bibliometric study focused on the electronically registered production between 1969 \\ and 2018, using descriptive statistical analysis. \\ Results: 213 articles were retrieved, published in 123 journals. Nursing contributed with 21 \\ (9.8\%) articles in the health area. The United States led the ranking with 92 articles $(43.1 \%)$. \\ The study shows the limitations of the WebQualis journal ranking, showing that 41 (33.36\%) \\ journals are not part of any Qualis level. \\ Conclusion: the data elaborated in this study measured the contribution of the scientific \\ knowledge deriving from the publications about catheterization. Therefore, its indicators can \\ be useful for the planning and execution of further studies, and for the scientific community's \\ knowledge on the system it is part of.
}

DESCRIPTORS: Nursing; Bibliometrics; Impact Factor; Catheterization, Peripheral; Pediatrics.

\section{ANÁLISIS BIBLIOMÉTRICO DE ARTÍCULOS SOBRE CATETERISMO VENOSO PERIFERICO EN PEDIATRIA}

\section{RESUMEN}

Objetivo: analizar la producción intelectual sobre cateterismo venoso periférico pediátrico, disponible en la base Scopus.

Método: estudio bibliométrico de la producción registrada electrónicamente de 1969 a 2018, con análisis estadístico descriptivo.

Resultados: fueron recuperados 213 artículos publicados en 123 periódicos. La Enfermería contribuyó con $21(9,8 \%)$ artículos del área de Salud. Los Estados Unidos lideraron con 92 artículos $(43,1 \%)$. El estudio muestra las limitaciones de la estratificación de periódicos por el WebQualis, demostrando que 41 (33,36\%) periódicos no hacen parte de ningún estrato Qualis.

Conclusión: los datos elaborados mediante el estudio mensuraron la contribución del conocimiento científico derivado de las publicaciones acerca de la cateterización. Por lo tanto, sus indicadores pueden ser útiles para la planificación y la ejecución de nuevas investigaciones, y para el conocimiento de la comunidad científica sobre el sistema en que está insertada.

DESCRIPTORES: Enfermería; Bibliometría; Factor de Impacto; Cateterismo Periférico; Pediatría. 
A hospitalização é, para o senso comum, uma condição que provoca alterações diretas na vida do ser humano, seja pelo fato iminente de que o motivo seja o adoecimento, mas também pela ruptura com suas necessidades sociais, principalmente o afastamento de seus familiares, sendo este último mais devastador quando se trata de crianças ${ }^{(1)}$.

Na pragmática assistencial, os profissionais de enfermagem se dedicam em oferecer aos seus pacientes atenção, orientações, avaliação física e outros cuidados a partir de uma variedade de técnicas e procedimentos. Estes são realizados em virtude da condição clínica e necessidades individuais, não raramente, procedimentos com níveis de invasão do corpo, mormente causadores de dor e sofrimento para o paciente e também seus acompanhantes, como no caso do cuidado em enfermagem em pediatria ${ }^{(1)}$.

É razoável admitir que a cateterização venosa periférica (CVP) é o procedimento mais comum vivenciado por pacientes e profissionais de enfermagem em ambiente hospitalar e poderia se configurar uma necessidade corriqueira se não fossem considerados dois aspectos importantes: a via de acesso e a idade do paciente que será submetido a este procedimento.

Esta propositura destaca a CVP em pediatria como procedimento realizado repetidas vezes por ocasião da condição clínica da criança, somada à sua capacidade cognitiva e motora, para prolongar o tempo de acesso venoso, em que pese o fato da presença à beira do leito de um acompanhante, que via de regra é a sua mãe ${ }^{(2)}$.

Para as crianças, a CVP representa um momento de dor física, psíquica, de sofrimento e estresse, situações estas que poderão impactar diretamente o tratamento, e não obstante, a relação envolvendo o trinômio - criança/acompanhante/enfermagem. Há de se considerar ainda o ambiente vivido(2) que permeia esta relação, ambiente muitas vezes desconhecido pela criança e pela mãe ou ainda ambiente rememorado por ambos, como no caso de internações anteriores.

Pesquisadores consideram que crianças de dois a sete anos são as mais vulneráveis aos efeitos não só da hospitalização como também das experiências dolorosas, porque elas apresentam estrutura cognitiva e psicomotora em desenvolvimento, daí se explica a dificuldade dessa faixa etária na hora de ser submetida a tal procedimento(3).

Em decorrência dos fatores intervenientes da execução da CVP estarem no plano da multidisciplinaridade, uma vez que envolvem aspectos da Psicologia, Sociologia, Biologia, Física, Anatomia entre outros, é possível que pesquisadores de diferentes áreas do conhecimento tenham dirigido suas pesquisas para buscar e propor maneiras de intervir na prática da cateterização venosa periférica em crianças. Portanto, o estado da arte de tais pesquisas poderá se beneficiar em decorrência de estudos bibliométricos como este.

Os estudos bibliométricos estão embasados em um conjunto de leis e princípios empíricos, provenientes da ciência da informação, cujo objetivo é investigar os aspectos quantitativos da produção, da disseminação e do uso da informação disponível e registrada, contribuindo, dessa forma, para a avaliação do estado atual da ciência, assim como do gerenciamento da pesquisa ${ }^{(5-7)}$.

A análise bibliométrica, que compreende na aplicação da estatística à bibliografia, possui três leis classicamente reconhecidas: Lei Bradford (lei de dispersão do conhecimento científico), Lei de Lotka (lei de produtividade de autores) e Lei de Zipf (frequência de palavras). Vale destacar que a principal diferença entre bibliografia e bibliometria é que esta última utiliza principalmente métodos quantitativos em relação a discursivos, o que confere maior objetividade na avaliação da produção científica(4). Contudo, a bibliometria não se preocupa somente com o aspecto quantitativo, mas também em verificar a relevância e o impacto de autores, periódicos, instituições, grupos ou países nas mais diversas áreas do saber ${ }^{(4-5)}$. 
Esta pesquisa justifica-se por investigar as características da produção intelectual acerca da temática cateterização venosa periférica em pediatria, podendo contribuir para outros pesquisadores da saúde ou de áreas associadas, pois mostrará a distribuição da produção no tempo, por área geográfica e do conhecimento, o impacto dos periódicos, os autores mais produtivos, entre outros.

Mesmo com um grande volume de livros de Enfermagem e de Medicina que abordam a CVP, inclusive na Pediatria, há pouca informação sobre o atual estado da arte das pesquisas acadêmicas relacionadas a este tema, fato que remete o estudo a investigar a seguinte questão: o nível de produtividade de autores e periódicos sobre cateterização venosa periférica pediátrica está correlacionada às leis e princípios bibliométricos da produção intelectual de autoria e publicação?

Com a finalidade de responder a esta questão por meio da utilização de métricas bibliométricas, tem-se como objetivo analisar a produção intelectual sobre cateterização venosa periférica pediátrica, disponibilizada na base de dados Scopus.

\section{MÉTODO}

Trata-se de um estudo bibliométrico que abordou a produção/disseminação e o uso da informação registrada eletronicamente em base de dados internacional, publicada entre 1969 e 2018. O material de análise foi limitado a artigos indexados na base Scopus, para tanto, utilizou-se como critério de inclusão: artigos de pesquisa e de revisão publicados entre 1969 e 2018, excluindo-se artigos com escopos diferentes. O princípio da bibliometria compreende o uso de indicadores confiáveis, que podem ser definidos como parâmetros utilizados em processos de avaliação(5).

As buscas foram realizadas na base de dados da Scopus no mês de abril de 2018, utilizando-se os descritores [catheterization], [peripheral] e [pediatrics] e como filtro "título do artigo, resumo e palavras-chave".

A escolha da base Scopus se deve por sua aceitação na comunidade científica nacional e internacional, sobretudo para a área da saúde, a qual disponibiliza resumos e citações de literatura científica revisada por pares, além de oferecer uma visão mais abrangente sobre a produção de pesquisa do mundo.

A análise descritiva da distribuição dos periódicos e autoria foi processada pelo programa $R \otimes$, considerando-se um nível de significância de $5 \%(p$ valor $=0,05)$. Foi realizada análise bivariada para comparar o comportamento bibliométrico dos periódicos, suas áreas de publicação e estratificação do Qualis. O programa VOSViewers foi utilizado para calcular a força dos links estabelecidos entre autores e coautores, bem como de áreas geográficas, representadas em forma de mapa.

O teste do qui-quadrado e o teste Shapiro-Wilk foram empregados na comparação de proporções, conforme o número de periódicos e para o teste de normalidade. Para as variáveis quantitativas, utilizaram-se a análise de variância (ANOVA) one-way, seguida pelo teste de Kuskal-Wallis.

\section{RESULTADOS}

A busca retornou com 213 documentos publicados por 160 autores e 875 coautores, dos quais $156(73,2 \%)$ foram artigos originais, $37(17,3 \%)$ revisões e $18(9,5 \%)$ outras modalidades.

A média de autores por artigo foi de 1,3. O autor mais producente publicou quatro artigos, enquanto que a maioria, 131 autores $(81,8 \%)$, publicaram um artigo. A distribuição 
geográfica das publicações recuperadas demonstra a liderança absoluta dos Estados Unidos da América com 92 documentos (43,1\%), seguido pelo Canadá com $17(7,9 \%)$ publicações, estando o Brasil no 12o lugar com quatro artigos (1,8\%). Os outros 32 países publicaram juntos 100 artigos (46,95\%). A análise geográfica de coautoria identificou 36 países. A distribuição considerou, apenas, países com no mínimo dois artigos publicados, o que resultou em menos da metade dos países (15), os 21 restantes não publicaram mais do que um artigo.

O idioma das publicações está concentrado na língua inglesa, com 196 (92\%) artigos, seguidos pelo Francês sete $(3,2 \%)$, Espanhol seis $(2,8 \%)$, Alemão um $(0,46 \%)$ e Português três $(1,54 \%)$.

Os 213 artigos foram produzidos por 79 instituições, sendo 69 (87,3\%) universidades e $10(12,7 \%)$ instituições hospitalares. A universidade que mais produziu foi a University of Toronto, com seis $(2,81 \%)$ artigos. Em seguida, a University of Pennsylvania com cinco (2,34\%). As universidades University of Tennessee Health Science Center, Mount Sinai Hospital of University of Toronto e University of Connecticut, publicaram, igualmente, quatro $(1,87 \%)$ artigos. Oito instituições publicaram três $(1,40 \%)$ artigos, 24 publicaram dois $(0,93 \%)$ artigos, 43 (54,43\%) instituições um $(0,46 \%)$ artigo.

A Enfermagem contribuiu com $21(9,8 \%)$ artigos da área de Saúde, atrás apenas da Medicina com 83 artigos (38,9\%). No que concerne à Área de conhecimento/Qualis (Gráfico 1) e $h$ Index/Área (Gráfico 2), é possível observar que os periódicos estratificados pela Enfermagem estão concentrados em A1 e A2, enquanto que na Medicina, em B2 e B1. O teste de Shapiro-Wilk revelou p valor $>0,05$, portanto, a amostra segue distribuição normal. O teste Kruskal-Wallis foi utilizado para avaliar a distribuição nas variáveis $h$ index e Área, resultando em p-valor >0,05, ou seja, possuem a mesma distribuição.

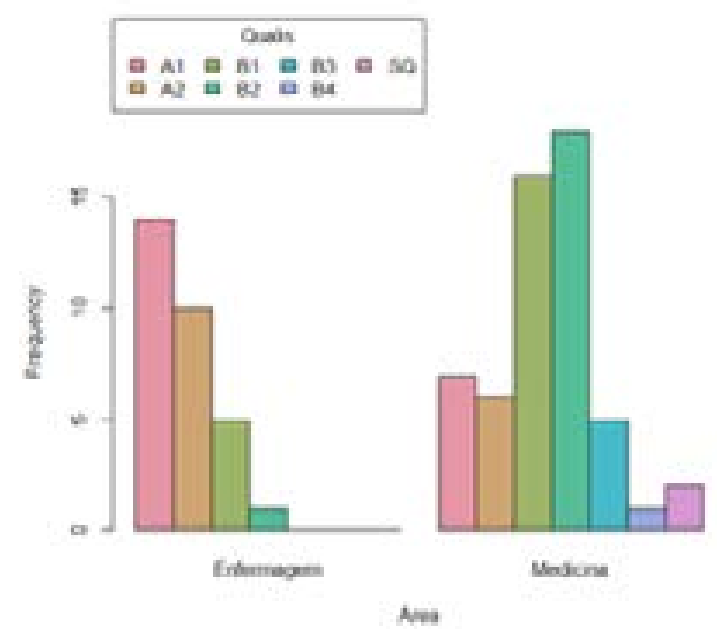

Gráfico 1 - Distribuição de periódicos por extrato Qualis. Rio de Janeiro, RJ, Brasil, 2018 


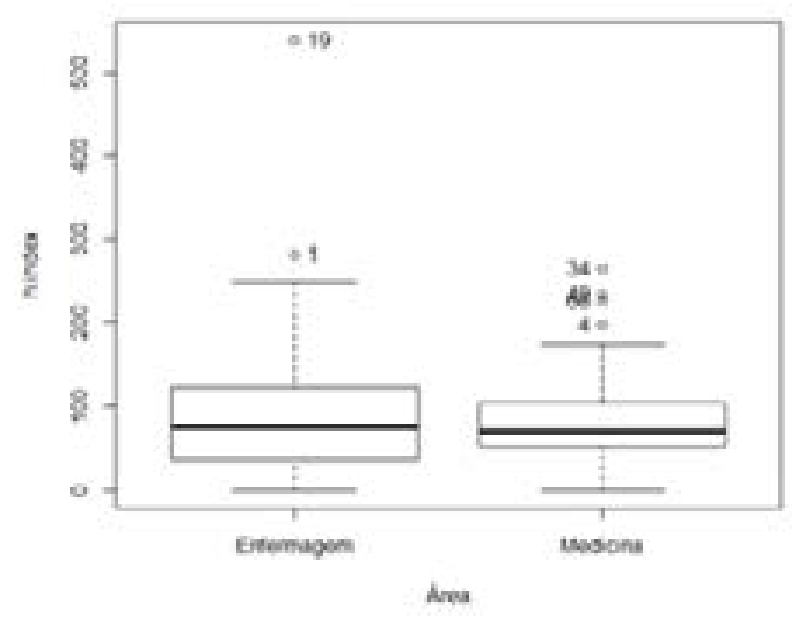

Gráfico 2 - Distribuição de periódicos por $h$ index/Área. Rio de Janeiro, RJ, Brasil, 2018

Os 213 documentos encontrados estão distribuídos em 123 periódicos, com média de 1,7 artigos por periódico. A revista Pediatrics publicou 30 (14\%) documentos, o que comprova sua significante contribuição para o tema, visto que 53 periódicos $(42,7 \%)$ publicaram um documento. As médias, medianas e desvios padrão de periódicos nas Áreas de Enfermagem, Medicina e SQ, foram, sucessivamente: médias - 66.43333, 57.34545, 65.23684; medianas: 66.5, 54.0, 68.5; desvios padrão - 38.39691, 36.87728, 31.49040.

As médias, medianas e desvios padrão de periódicos nos estratos A1, A2, B1, B2, B3, B4 e SQ foram, sucessivamente: médias - 53.95238, 72.12500, 68.57143, 56.78947, 56.80000, 50.00000 e 62.15000; medianas - 52.0, 86.5, 66.0, 41.0, 37.0, 50.0 e 63.5; desvios padrão - 32.69629, 36.96643, 35.63085, 38.43115, 56.05979 e NA 33.56322.

A Tabela 1 apresenta de forma resumida a Tabela de Bradford com distribuição dos periódicos e sua produção. Ela foi construída considerando o número de periódicos ( $N$. Periódicos) necessário para publicar uma quantidade determinada de artigos (N. Artigos).

Tabela 1 - Tabela de Bradford resumida. Rio de Janeiro, RJ, Brasil, 2018

\begin{tabular}{cccc}
$\mathbf{R}^{*}$ & N. Periódicos & N. Artigos & $\boldsymbol{\Sigma}$ Parcial \\
\hline 1 & 1 & 30 & 30 \\
\hline 2 & 1 & 6 & 36 \\
\hline 3 & 4 & 20 & 56 \\
\hline 4 & 5 & 20 & 76 \\
\hline 5 & 4 & 12 & 88 \\
\hline 6 & 17 & 34 & 122 \\
\hline 7 & 91 & 91 & 123 \\
\hline Total & 123 & 213 & 123
\end{tabular}

*equivalente à ordem de série 
A Tabela 2 apresenta a comparação do cálculo teórico com os achados empíricos. A produção dos periódicos foi dividida em três Zonas, cada qual contendo $1 / 3$ do total de 213 artigos, portanto, Y que representa o número de periódicos da $1^{\text {a }}$ Zona foi igual a 9.

Tabela 2 - Cálculo teórico e empírico de Bradford. Rio de Janeiro, RJ, Brasil, 2018

Cálculo Teórico

\begin{tabular}{cccccc}
\hline Zonas & $\begin{array}{c}\text { Total de } \\
\text { artigos }\end{array}$ & N periódicos & $\begin{array}{c}\text { Total de } \\
\text { artigos }\end{array}$ & N periódicos & Em linha \\
\hline $1^{\text {a }}$ & 71 & 9 & 69 & 9 & Não \\
\hline $2^{\text {a }}$ & 71 & 27 & 71 & 40 & Não \\
\hline $3^{\text {a }}$ & 71 & 243 & 73 & 74 & Não
\end{tabular}

\section{DISCUSSÃO}

Após estas análises, pode-se comprovar que o corpus da pesquisa é consistente, pois a mineração dos dados permitiu a recuperação dos documentos a partir dos principais descritores, delimitando o assunto que foi o foco da pesquisa.

Observou-se aumento sustentável de publicações a partir de 2002, sendo o ano de 2013 o que teve o maior número de publicações, mas ainda assim, a produtividade dos periódicos foi muito irregular e dispersa.

Foi possível verificar também baixa produtividade, em contrapartida, a média de coautoria mostrou-se dentro da média admitida na maioria dos periódicos nacionais e internacionais, de no máximo seis autores por artigo.

É bastante razoável inferir que o baixo índice de coautoria se deve, provavelmente, à baixa relevância do assunto nas publicações científicas. Esta constatação é reforçada quando se buscou a identificação de um possível grupo de Elite na produção de artigos sobre o tema/assunto. Nesse aspecto, para que haja um grupo de Elite estabelecido, a Lei do Elitismo(5) determina que os membros pertencentes a este grupo deverá produzir, no mínimo, 50\% das publicações, o que não ocorreu neste estudo.

Neste caso, a não determinação de um Grupo de Elite nos dados empíricos, decorre do elevado número de autores ocasionais ${ }^{(6)}$, podendo indicar fortemente fraca consolidação do tema com a área específica da pediatria ou estado de obsolescência.

A análise de coautoria possibilita a verificação da colaboração científica que é um dos atributos mais pesquisados na utilização da Análise de Redes Sociais (ARS), pois proporciona ao pesquisador visão ampla dos colégios invisíveis nos quais os vértices da pesquisa estão imersos, além de uma série de outras constatações quanto às relações de união no âmbito científico(6-7).

Neste aspecto, quando comparamos a média de coautoria com as instituições e países de origem, é possível constatar que as Redes Sociais no âmbito científico estão limitadas entre autores cuja filiação é a mesma em sua grande maioria, excetuando-se os Estados Unidos da América, Reino Unido, Canadá e Alemanha, que juntos, formaram a maior rede de colaboração interpaíses, seguidos por Austrália e França. 
O idioma das publicações foi dominado pelo Inglês. Esta dominância pode ser facilmente explicada, por ser o inglês um idioma universal para a ciência.

O Brasil aparece como o único da América Latina que, a exemplo de outros países, não possui redes e está totalmente isolado. Esta constatação permite inferir com segurança que o nível de interação internacional dos pesquisadores brasileiros, e os dos demais países que não formaram redes/links ${ }^{(7)}$, mesmo após 49 anos - 1969-2018, permanece ainda muito frágil.

A média de documentos publicados por instituição foi baixa quando se considera o recorte temporal estudado e outros estudos na área de enfermagem ${ }^{(3)}$. Portanto, não houve uma instituição que se destacasse quantitativamente.

Aplicando a Lei de Bradford para verificar o comportamento da distribuição/dispersão dos periódicos, cujo enunciado é: ao construir uma tabela em ordem decrescente da produção dos periódicos sobre um determinado tema, será possível distinguir um núcleo de periódicos mais vocacionados ao tema estudado e vários grupos/zonas com o mesmo número de artigos que núcleo, porém, com maior quantidade de periódicos na proporção de: $($ Zona $1=\mathrm{Y})$, (Zona $2=3 \mathrm{Y})$ e (Zona $\left.3=3 \mathrm{Y}^{2}\right)$.

A partir das Tabelas 1 e 2, foi possível identificar que em nenhuma das três Zonas, os dados empíricos são compatíveis e, portanto, em linha com o comportamento do modelo teórico de Bradford, apenas a primeira Zona apresentou uma aproximação maior. Esta Zona comportou 69 artigos em nove periódicos, enquanto que o modelo teórico determinou 71 artigos em 9 periódicos, sendo estes os mais devotados para publicação aceca do assunto. O Título Pediatrics foi o mais produtivo, comprovando sua especificidade sobre o assunto CVP.

A segunda Zona de Bradford não está em linha com o modelo teórico utilizado, o qual prevê um comportamento de distribuição de 71 artigos em 27 periódicos. O mesmo ocorreu com a terceira Zona, pois, deveria abrigar 71 artigos em 243 periódicos, no entanto, o comportamento empírico está fora de linha ${ }^{(7)}$ comportando 73 artigos em 74 periódicos.

Uma quantidade significativa de periódicos não se encontra estratificada no WebQualis, no entanto, são periódicos com SJR (Scimago Journal Report) significativos para a área de saúde, como é o caso do periódico Anaesthesia, terceiro lugar no ranking de periódicos mais devotados ao assunto estudado, portanto, um veículo científico importante, mas que ainda não recebeu artigo oriundo de programas de pós-graduação stricto sensu brasileiro e, por isso, não foi ainda estratificado.

Não foram encontrados periódicos nos estratos B5 e C. Esses dados mostram que, apesar da baixa produtividade, os artigos estão publicados em periódicos bem avaliados, - que pode levantar a hipótese de que a baixa quantidade de artigos sobre CVP pode estar relacionada à baixa qualidade dos artigos, ou seja, eles são submetidos, mas não são recomendados para publicação.

Considerando o tempo percorrido de registro do tema na base Scopus, é possível inferir que o assunto já atingiu sua maturidade ${ }^{(6-7)}$ e se encontra em estado de obsolescência de produção, talvez por não possuir mais o interesse da comunidade científica e/ou dos periódicos, em que pese o fato de alguns serem extremamente específicos, no entanto, não apresentam produção em quantidade considerável que possa demonstrar a importância/ relevância do assunto.

\section{CONCLUSÃO}

Considera-se que títulos e resumos de artigos são relevantes como representativos na produção científica acerca do tema. Os resultados apresentam baixa produção de estudos, distribuídos pelas mais variadas áreas de conhecimento, não se restringindo à Saúde. 
As potencialidades da utilização da Bibliometria podem ser consideradas um tratamento metodológico informacional sobre a produção do conhecimento nas diversas áreas. A Bibliometria contribuiu para estudar o registro da produção. A estruturação de bases de dados facilitou a obtenção dos mesmos e os programas disponíveis no mercado por sua vez agilizam o tratamento e a análise quantitativa de tais dados.

Os pesquisadores em enfermagem podem se beneficiar das técnicas de análise bibliométricas, não apenas em uma abordagem quantitativa, mas também qualitativa, pois os números auxiliam na leitura aproximada da realidade e, com a inclusão de estudos mais aprofundados, a riqueza das análises torna-se mais representativa. Portanto, os estudos bibliométricos podem contribuir para a visualização das conexões entre informações de diversas áreas do conhecimento.

Quanto aos autores, evidenciou-se a quantidade inexpressiva de autores por artigo e, quanto aos mais produtivos, o estudo destacou o periódico que veiculou suas pesquisas, a instituição ao qual estão vinculados e sua localização geográfica, neste particular, fica evidente que o assunto parece não despertar o interesse de pesquisadores ao ponto de constituírem redes de colaboração interpaíses e interinstituições.

Muito embora baseada em fatos empíricos, as leis de Bradford e Lotka conseguiram neste estudo, confirmar hipóteses de que o núcleo de periódicos é formado pelos mais devotados e, portanto, mais produtivos. Não obstante, revelou que, quanto mais específico for o assunto/tema, mais limitada será a possibilidade de identificação de grupos de Elite de autores.

O estudo mostra as limitações da estratificação de periódicos pelo WebQualis ao evidenciar que 41 periódicos (33,36\%) não constam em nenhum estrato Qualis, no entanto, são veículos com alto fator de impacto mensurados por duas métricas distintas, SJR e $h$ index.

Outro aspecto importante e que precisa ser considerado, refere-se à institucionalização cognitiva das áreas de conhecimento de domínio dos artigos e periódicos recuperados, que pôde ser identificada pelo uso dos três descritores utilizados. Em que pese a padronização gramatical dos descritores por um vocabulário controlado - DECs, ainda assim a realização de inferências não confiáveis pode se configurar em uma limitação deste estudo.

Como contribuição, os resultados do estudo e seu desenho metodológico poderão servir de base para outras proposições, preenchendo assim, uma lacuna no rol de pesquisas bibliométricas em enfermagem, como o compromisso de consolidar seu corpo de conhecimento, desenvolvendo pesquisas que possa contribuir simultaneamente para o crescimento e consolidação desse saber.

\section{REFERÊNCIAS}

1. Gomes GLL, Fernandes MGM, da Nóbrega MML. Ansiedade da hospitalização em crianças: análise conceitual. Rev. bras. enferm. [Internet]. 2016 [acesso em 01 maio 2018]; 69(5). Disponível em: http:// dx.doi.org/10.1590/0034-7167-2015-0116.

2. Almeida TJC de, Miranda JOF, Santos LM dos, Santana RCB de, Camargo CL de, Nascimento Sobrinho CL. Peripheral venous accesses in hospitalized children: a photographic study. Rev. enferm. UFPE on line. [Internet]. 2016 [acesso em 01 maio 2018]; 10 (Suppl 2). Disponível em: https://periodicos.ufpe.br/ revistas/revistaenfermagem/article/view/11009.

3. Motta AB, Perosa GB, Barros L, Silveira KA, Lima ASS, Carnier LE, et al. Comportamentos de coping no contexto da hospitalização infantil. Estud. psicol. [Internet]. 2015 [acesso em 01 maio 2018]; 32(2).

Disponível em: http://dx.doi.org/10.1590/0103-166X2015000200016.

4. Marcias-Chapula CA. O papel da informetria e da cienciometria e sua perspectiva nacional e 
internacional. Ci. Inf. [Internet]. 1998 [acesso em 01 maio 2018]; 27 (2). Disponível em: http://dx.doi. org/10.1590/S0100-19651998000200005.

5. Araújo CAA. Bibliometria: evolução histórica e questões atuais. Em Questão [Internet]. 2006 [acesso em 01 maio 2018]; 15(4). Disponível em: https://seer.ufrgs.br/EmQuestao/article/view/16.

6. Barreto ML. O desafio de avaliar o impacto das ciências para além da bibliometria. Rev. Saúde Públ. [Internet]. 2013 [acesso em 01 maio 2018]; 47(4). Disponível em: http://dx.doi.org/10.1590/S0034$\underline{8910.2013047005073 .}$

7. Medeiros JMG de, Vitoriano MAV. A evolução da bibliometria e sua interdisciplinaridade na produção científica brasileira. Rev. Digit. Bibliotecon. E Cienc. Inf. [Internet]. 2015 [acesso em 01 maio 2018]; 13(3). Disponível em: http://dx.doi.org/10.20396/rdbci.v13i3.8635791.

Recebido: 01/05/2018

Finalizado: 25/02/2019

\section{Autor Correspondente:}

Viviane Reis Fontes da Silva

Universidade Federal do Estado do Rio de Janeiro

Av. Pasteur, 296 - 22290-250 - Rio de Janeiro, RJ, Brasil

E-mail: vivianereisfontes@gmail.com

\section{Contribuição dos autores:}

Contribuições substanciais para a concepção ou desenho do estudo; ou a aquisição, análise ou interpretação de dados do estudo - VRFS, IBM, CC, RCLS, CRLS, LCS

Elaboração e revisão crítica do conteúdo intelectual do estudo - CRLS

Aprovação da versão final do estudo a ser publicado - CRLS, LCS 